\title{
Space-time fractional Rosenou-Haynam equation: Lie symmetry analysis, explicit solutions and conservation laws
}

\author{
Dumitru Baleanu ${ }^{1,2^{*}}$, Mustafa Inc ${ }^{3}$, Abdullahi Yusuf $^{3}$ and Aliyu Isa Aliyu ${ }^{3}$
}

\section{"Correspondence:}

dumitru@cankaya.edu.tr

'Department of Mathematics,

Cankaya University, Öğretmenler

Cad., Ankara, 1406530, Turkey

${ }^{2}$ Institute of Space Sciences,

Magurele, Bucharest, Romania

Full list of author information is

available at the end of the article

\section{Springer}

\begin{abstract}
This article uses the extension of the Lie symmetry analysis (LSA) and conservation laws (Cls) (Singla et al. in Nonlinear Dyn. 89(1):321-331, 2017; Singla et al. in J. Math. Phys. 58:051503, 2017) for the space-time fractional partial differential equations (STFPDEs) to analyze the space-time fractional Rosenou-Haynam equation (STFRHE) with Riemann-Liouville (RL) derivative. We transform the space-time fractional RHE to a nonlinear ordinary differential equation (ODE) of fractional order using its Lie point symmetries. The reduced equation's derivative is in Erdelyi-Kober (EK) sense. We use the power series (PS) technique to derive explicit solutions for the reduced ODE for the first time. The Cls for the governing equation are constructed using a new conservation theorem.
\end{abstract}

Keywords: space-time fractional RHE; Lie symmetry analysis; RL fractional derivative; explicit solutions; $\mathrm{Cls}$

\section{Introduction}

Fractional differential equations (FDEs) are generalizations of classical differential equations of integer order. FDEs have been studied nowadays to describe several physical aspects and procedure in natural conditions [3-19]. However, fractional partial differential equations (FPDEs) having only time derivative have been analyzed via the Lie symmetry method [20-26]. Recently, the Lie method has been developed to the systems of time FPDEs [27]. More recently, the Lie method has been extended for the first time to the analysis of FPDEs having space and time derivative with fractional order and the systems of space and time FPDEs in [1,2]. To the best of our knowledge, application of the Lie method to the space-time FPDEs and the systems of space-time FPDEs appeared only in $[1,2]$. Therefore, applying this new approach to more space-time FPDEs in order to obtain lots of solutions will be remarkable contribution to the literature.

The LSA and Cls supply lots of ideas on the systems modeled by the differential equations. Lie symmetries are a very significant tool in determining exact solutions and Cls of differential equations. In spite of the significance of $\mathrm{Cls}$ in analyzing the integrability and internal properties and in proving the existence and uniqueness of solutions of differential equations [28-30], the Cls for PDEs having fractional-order are not investigated in detail. The generalization for investigating Cls for FDEs was presented in [31,32]. In recent time,

(c) The Author(s) 2018. This article is distributed under the terms of the Creative Commons Attribution 4.0 International License (http://creativecommons.org/licenses/by/4.0/), which permits unrestricted use, distribution, and reproduction in any medium, provided you give appropriate credit to the original author(s) and the source, provide a link to the Creative Commons license, and indicate if changes were made. 
the fractional generalized Noether operators have been introduced [30] for FPDEs that do not have a Lagrangian in order to find Cls using a new conservation theorem [33]. Even though very few works [34-36] that have to do with Cls of FPDEs can be found, the analysis of Cls for the STFPDEs has entirely not been investigated in more detail.

In this study, we consider STFRHE given by

$$
\frac{\partial^{\alpha} u}{\partial t^{\alpha}}+u \frac{\partial^{\beta} u}{\partial x^{\beta}}+u u_{x x x}+3 u_{x} u_{x x}=0
$$

where $0<\alpha \leq 1$ and $\beta<2$. In Eq. (1), $\frac{\partial^{\alpha} u}{\partial t^{\alpha}}$ and $\frac{\partial^{\beta} u}{\partial x^{\beta}}$ are the RL fractional derivatives of order $\alpha, \beta>0$, respectively. If $\beta=1$, Eq. (1) becomes

$$
\frac{\partial^{\alpha} u}{\partial t^{\alpha}}+u \frac{\partial u}{\partial x}+u u_{x x x}+3 u_{x} u_{x x}=0
$$

The invariance properties for Eq. (2) that can be used to interpret the formation of patterns in liquid drops were analyzed and investigated in [37]. If $\alpha=1$ and $\beta=1$, Eq. (1) becomes

$$
\frac{\partial u}{\partial t}+u \frac{\partial u}{\partial x}+u u_{x x x}+3 u_{x} u_{x x}=0
$$

Some approximate analytical solutions for Eq. (3) were presented in [38, 39]. Many methods have been applied to reach analytical solutions for PDEs [40-47].

The aim of this study is to analyze and investigate the LSA, explicit solution via the power series technique and Cls for Eq. (1).

The outline of the paper is presented in the following way: In Section 2 we present some preliminaries; in Section 3 we present symmetry analysis and reduction; in Section 4 we analyze explicit solution for the reduced equation; in Section 5 we construct Cls for the underlying equation. Finally, concluding remarks are given in Section 6.

\section{Preliminaries}

Herein, we present some preliminaries. The RL fractional derivative $[19,27]$ is defined by

$$
D^{\alpha} f(t)= \begin{cases}\frac{d^{n} f}{d t^{n}}, & \alpha=n, \\ \frac{d}{d t^{n}} I^{n-\alpha} f(t), & 0 \leq n-1<\alpha<n,\end{cases}
$$

where $n$ is a natural number and $I^{\mu} f(t)$ is given by

$$
\begin{aligned}
& I^{\mu} f(t)=\frac{1}{\Gamma(\mu)} \int_{0}^{t}(t-s)^{\mu-1} f(s) d s, \quad \mu>0, \\
& I^{\mu} f(t)=f(t),
\end{aligned}
$$

and $\Gamma(z)$ represents the gamma function.

Consider the following space-time FPDEs:

$$
\Delta=F\left(x, t, u, \partial_{t}^{\alpha}, \partial_{x}^{\beta}, u_{x x}, u_{x x x}, \ldots\right) .
$$


Suppose that a one-parameter group of transformations is given by

$$
\begin{aligned}
& \bar{t}=t+\epsilon \xi^{2}(t, x, u)+O\left(\epsilon^{2}\right), \\
& \bar{x}=x+\epsilon \xi^{1}(t, x, u)+O\left(\epsilon^{2}\right), \\
& \bar{u}=u+\epsilon \eta(t, x, u)+O\left(\epsilon^{2}\right), \\
& \frac{\partial^{\alpha} \bar{u}}{\partial \bar{t}}=\frac{\partial^{\alpha} u}{\partial t^{\alpha}}+\epsilon \eta^{\alpha, t}(t, x, u)+O\left(\epsilon^{2}\right), \\
& \frac{\partial^{\beta} \bar{u}}{\partial \bar{x}}=\frac{\partial^{\beta} u}{\partial x^{\beta}}+\epsilon \eta^{\beta, x}(t, x, u)+O\left(\epsilon^{2}\right), \\
& \frac{\partial \bar{u}}{\partial \bar{x}}=\frac{\partial u}{\partial x}+\epsilon \eta^{x}(t, x, u)+O\left(\epsilon^{2}\right), \\
& \frac{\partial^{2} \bar{u}}{\partial \bar{x}^{2}}=\frac{\partial^{2} u}{\partial x^{2}}+\epsilon \eta^{x x}(t, x, u)+O\left(\epsilon^{2}\right), \\
& \frac{\partial^{3} \bar{u}}{\partial \bar{x}^{3}}=\frac{\partial^{3} u}{\partial x^{3}}+\epsilon \eta^{x x x}(t, x, u)+O\left(\epsilon^{2}\right),
\end{aligned}
$$

where

$$
\begin{aligned}
& \eta^{\alpha, t}=D_{t}^{\alpha}(\eta)+\xi^{1} D_{t}^{\alpha}\left(u_{x}\right)-D_{t}^{\alpha}\left(\xi^{1} u_{x}\right)+D_{t}^{\alpha}\left(u\left(D_{t} \xi^{2}\right)\right)-D_{t}^{\alpha+1}(\xi u)+\xi^{2} D_{t}^{\alpha+1}(u), \\
& \eta^{\beta, t}=D_{x}^{\beta}(\eta)+D_{x}^{\beta}\left(u\left(D_{x} \xi^{1}\right)\right)-D_{x}^{\beta+1}\left(\xi^{1} u\right)+\xi D_{x}^{\beta+1}(u)+\xi^{2} D_{t}^{\beta}\left(u_{t}\right)-D_{x}^{\beta}\left(\xi^{2} u_{t}\right) \\
& \eta^{x}=D_{x}(\eta)-u_{x} D_{x}\left(\xi^{1}\right)-u_{t} D_{t}\left(\xi^{2}\right) \\
& \eta^{x x}=D_{x}\left(\eta^{x}\right)-u_{x t} D_{x}\left(\xi^{1}\right)-u_{x x} D_{t}\left(\xi^{2}\right), \\
& \eta^{x x x}=D_{x}\left(\eta^{x x}\right)-u_{x x t} D_{x}\left(\xi^{1}\right)-u_{x x x} D_{t}\left(\xi^{2}\right) \\
& \ldots
\end{aligned}
$$

In Eq. (7), $D_{x}$ and $D_{t}$ represent the total differential operators defined by

$$
\begin{aligned}
& D_{x}=\frac{\partial}{\partial x}+u_{x} \frac{\partial}{\partial u}+u_{x x} \frac{\partial}{\partial u_{x}}+\cdots \\
& D_{t}=\frac{\partial}{\partial t}+u_{t} \frac{\partial}{\partial u}+u_{t t} \frac{\partial}{\partial u_{t}}+\cdots
\end{aligned}
$$

The corresponding Lie algebra of symmetries consists of the following vector fields:

$$
X=\xi^{1} \frac{\partial}{\partial x}+\xi^{2} \frac{\partial}{\partial t}+\eta \frac{\partial}{\partial u} .
$$

The vector field Eq. (12) is a Lie point symmetry of Eq. (7) provided

$$
\left.P^{\alpha, \beta, 3} r X(\triangle)\right|_{\triangle=0}=0
$$

Also, the invariance condition [48] yields

$$
\left.\xi^{1}(t, x, u)\right|_{x=0}=0,\left.\quad \xi^{2}(t, x, u)\right|_{t=0}=0,
$$

and the $\alpha$ th and $\beta$ th extended infinitesimals with Eq. (10) are given as in $[19,27]$. 


\section{Lie symmetries and reduction for Eq. (1)}

Suppose that Eq. (1) is an invariant under Eq. (8), we have that

$$
\frac{\partial^{\alpha} \bar{u}}{\partial \bar{t}^{\alpha}}+u \frac{\partial^{\beta} \bar{u}}{\partial \bar{x}^{\beta}}+\bar{u} \bar{u}_{\bar{x} \bar{x} \bar{x}}+3 \bar{u}_{\bar{x}} \bar{u}_{\bar{x} \bar{x}}=0
$$

so that $u=u(x, t)$ satisfies Eq. (1). Using Eq. (8) in Eq. (15), we get the invariant equation

$$
\eta^{\alpha, t}+u \eta^{\beta, x}+u_{x x x} \eta+3 u_{x x} \eta^{x}+3 u_{x} \eta^{x x}+u \eta^{x x x}=0
$$

Putting the values of $\eta^{\alpha, t}, \eta^{\beta, x}, \eta^{x}, \eta^{x x}$ and $\eta^{x x x}$ into Eq. (16) and isolating coefficients, we have

$$
\begin{aligned}
& \partial_{t}^{\alpha} \eta-u \partial_{t}^{\alpha} \eta_{u}+u \partial_{x}^{\beta} \eta-u^{2} \partial_{x}^{\beta} \eta_{u}+u \eta_{x x x}=0, \\
& \left(\begin{array}{l}
\alpha \\
n
\end{array}\right) \partial_{t}^{n}(\eta)-\left(\begin{array}{c}
\alpha \\
n+1
\end{array}\right) D_{t}^{n+1}\left(\xi^{2}\right)=0, \quad n=1,2, \ldots \\
& \left(\begin{array}{l}
\beta \\
n
\end{array}\right) \partial_{x}^{n}(\eta)-\left(\begin{array}{c}
\beta \\
n+1
\end{array}\right) D_{x}^{n+1}\left(\xi^{1}\right)=0, \quad n=1,2, \ldots, \\
& \xi_{u}^{1}=\xi_{u}^{2}=\xi_{t}^{1}=\xi_{x}^{2}=\eta_{u u}=0, \\
& u \beta \xi_{x}^{1}-\alpha \xi_{t}^{2}=0 .
\end{aligned}
$$

Solving these equations, we obtain

$$
\xi^{1}=c_{1}+x \alpha c_{2}, \quad \xi^{2}=t \beta c_{2}, \quad \eta=\alpha u c_{2}(3-\beta),
$$

where $c_{1}, c_{2}$ represent arbitrary constants. Thus infinitesimal symmetry group for Eq. (1) is as follows:

$$
X_{1}=\frac{\partial}{\partial x}, \quad X_{2}=\alpha x \frac{\partial}{\partial x}+\beta t \frac{\partial}{\partial t}+\alpha u(3-\beta) \frac{\partial}{\partial u} .
$$

In particular, for the symmetry $X_{2}$, we obtain the following similarity transformation and similarity variable:

$$
\frac{d x}{\alpha x}=\frac{d t}{t \beta}=\frac{d u}{\alpha u(3-\beta)} .
$$

Solving the above equations, we get

$$
z_{1}=x t^{-\frac{\alpha}{\beta}}, \quad z_{2}=u t^{-\frac{\alpha(3-\beta)}{\beta}} .
$$

Hence, from the symmetry $X_{2}$, we get the group-invariant solution

$$
u=t^{\frac{\alpha(3-\beta)}{\beta}} f(\xi), \quad \xi=x t^{-\frac{\alpha}{\beta}}
$$

where $f$ is an arbitrary function of $\xi$. 
Theorem By the similarity transformation, Eq. (19) reduces Eq. (1) to the following:

$$
\left(P_{\frac{\beta}{\alpha}}^{1-\alpha+\frac{\alpha}{\beta}(3-\beta), \alpha} f\right)(\xi)+f \xi^{-\beta}\left(Q_{1}^{-\beta, \beta} f\right)(\xi)+f f \xi \xi \xi+3 f_{\xi} f_{\xi \xi}=0
$$

with the left-hand-sided EK fractional differential operator $[49,50]$

$$
\begin{aligned}
& \left(P_{\delta}^{\xi^{2}, \alpha} f\right)=\prod_{j=0}^{n-1}\left(\xi^{2}+j-\frac{1}{\delta} \frac{d}{d \xi}\right)\left(K_{\delta}^{\xi^{2}+\alpha, n-\alpha} f\right)(\xi), \\
& n= \begin{cases}{[\alpha]+1,} & \alpha \neq \mathbf{N} \\
\alpha, & \alpha \in \mathbf{N}\end{cases}
\end{aligned}
$$

where

$$
\left(K_{\delta}^{\xi^{2}, \alpha} f\right)(\xi)= \begin{cases}\frac{1}{\Gamma(\alpha)} \int_{1}^{\infty}(u-1)^{\alpha-1} u^{-\left(\xi^{2}+\alpha\right)} f\left(\xi u^{\frac{1}{\delta}}\right) d u, & \alpha>0, \\ f(\xi), & \alpha=0 .\end{cases}
$$

The right-hand-sided EK fractional differential operator is given by

$$
\begin{aligned}
& \left(Q_{\delta}^{\xi^{2}, \beta} f\right)=\prod_{j=0}^{n-1}\left(\xi^{2}+j-\frac{1}{\delta} \frac{d}{d \xi}\right)\left(L_{\delta}^{\xi^{2}+\beta, n-\beta} f\right)(\xi), \\
& n= \begin{cases}{[\beta]+1,} & \beta \neq \mathbf{N} \\
\beta, & \beta \in \mathbf{N}\end{cases}
\end{aligned}
$$

where

$$
\left(L_{\delta}^{\xi^{2}, \beta} f\right)(\xi)= \begin{cases}\frac{1}{\Gamma(\beta)} \int_{0}^{1}(1-u)^{\beta-1} u^{\xi^{2}} f\left(\xi u^{\frac{1}{\delta}}\right) d u, & \beta>0, \\ f(\xi), & \beta=0 .\end{cases}
$$

Proof Let $n-1<\alpha<1, n=1,2,3, \ldots$. In accordance with the RL fractional derivative in Eq. (15), we get

$$
\frac{\partial^{\alpha} u}{\partial t^{\alpha}}=\frac{\partial^{n}}{\partial t^{n}}\left[\frac{1}{\Gamma(n-\alpha)} \int_{1}^{t}(t-s)^{n-\alpha-1} s^{\frac{\alpha(3-\beta)}{\beta}} f\left(x s^{-\left(\frac{\alpha}{\beta}\right)}\right) d s\right] .
$$

Let $v=\frac{t}{s}, d s=-\frac{t}{v^{2}} d v$. Thus, Eq. (27) becomes

$$
\frac{\partial^{\alpha} u}{\partial t^{\alpha}}=\frac{\partial^{n}}{\partial t^{n}}\left[t^{n-\alpha+\frac{\alpha(3-\beta)}{\beta}} \frac{1}{\Gamma(n-\alpha)} \int_{1}^{\infty}(v-1)^{n-\alpha-1} v^{-\left(n-\alpha+1+\frac{\alpha(3-\beta)}{\beta}\right)} f\left(\xi v^{\frac{\alpha}{\beta}}\right) d v\right] .
$$

Applying EK fractional integral operator Eq. (23) to Eq. (28), we get

$$
\frac{\partial^{\alpha} u}{\partial t^{\alpha}}=\frac{\partial^{n}}{\partial t^{n}}\left[t^{n-\alpha+\frac{\alpha(3-\beta)}{\beta}}\left(K_{\frac{\beta}{\alpha}}^{1+\frac{\alpha(3-\beta)}{\beta}, n-\alpha} f\right)(\xi)\right] .
$$

We simplify the right-hand side of Eq. (29). Considering $\xi=x t^{-\frac{\alpha}{\beta}}, \phi \in(0, \infty)$, we acquire

$$
t \frac{\partial}{\partial t} \phi(\xi)=t x\left(-\frac{\alpha}{\beta}\right) t^{-\frac{\alpha}{\beta}-1} \phi^{\prime}(\xi)=-\frac{\alpha}{\beta} \xi \frac{\partial}{\partial \xi} \phi(\xi) .
$$


Hence,

$$
\begin{aligned}
\frac{\partial^{n}}{\partial t^{n}} & \left.t^{n-\alpha+\frac{\alpha(3-\beta)}{\beta}}\left(K_{\frac{\beta}{\alpha}}^{1+\frac{\alpha(3-\beta)}{\beta}, n-\alpha} f\right)(\xi)\right] \\
= & \frac{\partial^{n-1}}{\partial t^{n-1}}\left[\frac{\partial}{\partial t}\left(t^{n-\alpha+\frac{\alpha(3-\beta)}{\beta}}\left(K_{\frac{\beta}{\alpha}}^{1+\frac{\alpha(3-\beta)}{\beta}, n-\alpha} f\right)(\xi)\right)\right] \\
= & \frac{\partial^{n-1}}{\partial t^{n-1}}\left[t^{n-\alpha+\frac{\alpha(3-\beta)}{\beta}-1}\left(n-\frac{\alpha}{\beta}-\frac{\alpha}{\beta} \xi \frac{\partial}{\partial \xi}\right)\right. \\
& \left.\times\left(K_{\frac{\beta}{\alpha}}^{1+\frac{\alpha(3-\beta)}{\beta}, n-\alpha} f\right)(\xi)\right] .
\end{aligned}
$$

Repeating $(n-1)$-times, we have

$$
\begin{aligned}
& \frac{\partial^{n}}{\partial t^{n}}\left[t^{n-\alpha+\frac{\alpha(3-\beta)}{\beta}}\left(K_{\frac{\beta}{\alpha}}^{1+\frac{\alpha(3-\beta)}{\beta}, n-\alpha} f\right)(\xi)\right] \\
&=\frac{\partial^{n-1}}{\partial t^{n-1}}\left[\frac{\partial}{\partial t}\left(t^{n-\alpha+\frac{\alpha(3-\beta)}{\beta}}\left(K_{\frac{\beta}{\alpha}}^{1+\frac{\alpha(3-\beta)}{\beta}, n-\alpha} f\right)(\xi)\right)\right] \\
&=\frac{\partial^{n-1}}{\partial t^{n-1}}\left[t^{n-\alpha+\frac{\alpha(3-\beta)}{\beta}-1}\left(n-\frac{\alpha}{\beta}-\frac{\alpha}{\beta} \xi \frac{\partial}{\partial \xi}\right)\right. \\
&\left.\quad \times\left(K_{\frac{\beta}{\alpha}}^{1+\frac{\alpha(3-\beta)}{\beta}, n-\alpha} f\right)(\xi)\right] \\
& \vdots \quad \\
&= t^{-\alpha+\frac{\alpha(3-\beta)}{\beta}} \prod_{j=0}^{n-1}\left[\left(1-\alpha+\frac{\alpha(3-\beta)}{\beta}+j-\frac{\alpha}{\beta} \xi \frac{\partial}{\partial \xi}\right)\right. \\
&\left.\quad \times\left(K_{\frac{\beta}{\alpha}}^{1+\frac{\alpha(3-\beta)}{\beta}, n-\alpha} f\right)(\xi)\right] .
\end{aligned}
$$

Applying EK fractional differential operator Eq. (21) to Eq. (33), we obtain that

$$
\frac{\partial^{n} u}{\partial t^{n}}\left[\left(t^{n-\alpha+\frac{\alpha(3-\beta)}{\beta}}\left(K_{\frac{\beta}{\alpha}}^{1+\frac{\alpha(3-\beta)}{\beta}, n-\alpha} f\right)(\xi)\right)\right]=t^{-\alpha+\frac{\alpha(3-\beta)}{\beta}}\left(P_{\frac{\beta}{\alpha}}^{1-\alpha+\frac{\alpha(3-\beta)}{\beta}, \alpha} f\right)(\xi) .
$$

Substituting Eq. (34) into Eq. (29), we have

$$
\frac{\partial^{\alpha} u}{\partial t^{\alpha}}=t^{-\alpha+\frac{\alpha(3-\beta)}{\beta}}\left(P_{\frac{\beta}{\alpha}}^{1-\alpha+\frac{\alpha(3-\beta)}{\beta}, \alpha} f\right)(\xi)
$$

Comparably, $\beta$ th-order with respect to $x$ can be derived to be the following:

$$
\frac{\partial^{\beta} u}{\partial x^{\beta}}=t^{\frac{\alpha(3-\beta)}{\beta}} \xi^{-\beta}\left(Q_{1}^{-\beta, \beta} f\right)(\xi)
$$

Thus, using Eqs. (35) and (36), we obtain

$$
\left(P_{\frac{\beta}{\alpha}}^{1-\alpha+\frac{\alpha}{\beta}(3-\beta), \alpha} f\right)(\xi)+f \xi^{-\beta}\left(Q_{1}^{-\beta, \beta} f\right)(\xi)+\not f \xi \xi \xi+3 f_{\xi} f_{\xi \xi}=0
$$

The proof of the theorem is completed. 


\section{Explicit power series solutions for Eq. (37)}

We investigate the explicit solutions via the PS method [51, 52]. Let

$$
f(\xi)=\sum_{n=0}^{\infty} a_{n} \xi^{n}
$$

From Eq. (38), we can have

$$
f^{\prime}=\sum_{n=0}^{\infty} n a_{n} \xi^{n-1}, \quad f^{\prime \prime}=\sum_{n=0}^{\infty} n(n-1) a_{n} \xi^{n-2}, \quad f^{\prime \prime}=\sum_{n=0}^{\infty} n(n-1)(n-2) a_{n} \xi^{n-3}
$$

Putting Eqs. (38) and (39) in Eq. (37), one obtains

$$
\begin{aligned}
& \sum_{n=0}^{\infty} \frac{\Gamma\left(2-\alpha+\frac{\alpha(3-\beta)}{\beta}-\frac{n \alpha}{\beta}\right)}{\Gamma\left(2+\frac{\alpha(3-\beta)}{\beta}-\frac{n \alpha}{\beta}\right)} a_{n} \xi^{n}+\sum_{n=0}^{\infty} a_{n} \xi^{n} \sum_{n=0}^{\infty} \frac{\Gamma(1-n)}{\Gamma(1-n+\beta)} a_{n} \xi^{n} \\
& \quad+\sum_{n=0}^{\infty} a_{n} \xi^{n} \sum_{n=0}^{\infty}(n+3)(n+2)(n+1) a_{n+3} \xi^{n} \\
& \quad+3 \sum_{n=0}^{\infty}(n+1) a_{n+1} \xi^{n} \sum_{n=0}^{\infty}(n+2)(n+1) a_{n+2} \xi^{n}=0 .
\end{aligned}
$$

Comparing coefficients in Eq. (40) when $n=0$, we get

$$
a_{3}=\frac{1}{6 a_{0}}\left(\frac{\Gamma\left(2-\alpha+\frac{\alpha(3-\beta)}{\beta}\right)}{\Gamma\left(2+\frac{\alpha(3-\beta)}{\beta}\right)} a_{0}+\frac{a_{0}^{2}}{\Gamma(1+\beta)}-6 a_{1} a_{2}\right),
$$

when $n \geq 1$, we have

$$
\begin{aligned}
a_{n+3}= & \frac{1}{(n+3)(n+2)(n+1)}\left\{\frac{\Gamma\left(2-\alpha+\frac{\alpha(3-\beta)}{\beta}+\frac{n \alpha}{\beta}\right)}{\Gamma\left(2+\frac{\alpha(3-\beta)}{\beta}-\frac{n \alpha}{\beta}\right)} a_{n}\right. \\
& +\sum_{k=0}^{n} \frac{\Gamma(1-n-k)}{\Gamma(1-n-k+\beta)} a_{k} a_{n-k} \\
& \left.-3 \sum_{k=0}^{n}(n-k+2)(n-k+1) a_{k} a_{n-k+2}\right\} .
\end{aligned}
$$

Thus, each coefficient $a_{n}(n \geq 1)$ for Eq. (38) is found by the arbitrary constants $a_{i}$ ( $i=$ $0,1,2)$. This means that the explicit PS solution for Eq. (37) exists, and its coefficients depend on Eqs. (41) and (42). Therefore, the PS solution for Eq. (37) can be represented in the form:

$$
\begin{aligned}
f(\xi) & =a_{0}+a_{1} \xi+a_{2} \xi^{2}+a_{3} \xi^{3} \sum_{n=1}^{\infty} a_{n+3} \xi^{n+3} \\
& =a_{0}+a_{1} \xi+a_{2} \xi^{2}+\frac{1}{6 a_{0}}\left(\frac{\Gamma\left(2-\alpha+\frac{\alpha(3-\beta)}{\beta}\right)}{\Gamma\left(2+\frac{\alpha(3-\beta)}{\beta}\right)} a_{0}+\frac{a_{0}^{2}}{\Gamma(1+\beta)}-6 a_{1} a_{2}\right) \xi^{3}
\end{aligned}
$$




$$
\begin{aligned}
& +\sum_{n=1}^{\infty} \frac{1}{(n+3)(n+2)(n+1)}\left\{\frac{\Gamma\left(2-\alpha+\frac{\alpha(3-\beta)}{\beta}+\frac{n \alpha}{\beta}\right)}{\Gamma\left(2+\frac{\alpha(3-\beta)}{\beta}-\frac{n \alpha}{\beta}\right)} a_{n}\right. \\
& +\sum_{k=0}^{n} \frac{\Gamma(1-n-k)}{\Gamma(1-n-k+\beta)} a_{k} a_{n-k} \\
& \left.-3 \sum_{k=0}^{n}(n-k+2)(n-k+1) a_{k} a_{n-k} a_{n-k+2}\right\} \xi^{n+3}
\end{aligned}
$$

Consequently, we acquire the explicit power series given by

$$
\begin{aligned}
u(x, t)= & a_{0} t^{\frac{\alpha(3-\beta)}{\beta}}+a_{1} x t^{\frac{\alpha(2-\beta)}{\beta}}+a_{2} x t^{\frac{\alpha(1-\beta)}{\beta}}+a_{3} x t^{-\alpha}+\sum_{n=1}^{\infty} a_{n+3} x t^{-\frac{\alpha(n+\beta)}{\beta}} \\
= & a_{0} t^{\frac{\alpha(3-\beta)}{\beta}}+a_{1} x t^{\frac{\alpha(2-\beta)}{\beta}}+a_{2} x t^{\frac{\alpha(1-\beta)}{\beta}} \\
& +\frac{1}{6 a_{0}}\left(\frac{\Gamma\left(2-\alpha+\frac{\alpha(3-\beta)}{\beta}\right)}{\Gamma\left(2+\frac{\alpha(3-\beta)}{\beta}\right)} a_{0}+a_{0}^{2} \xi^{-\beta} \Gamma(1-\beta)-6 a_{1} a_{2}\right) x t^{-\alpha} \\
& +\sum_{n=1}^{\infty} \frac{1}{(n+3)(n+2)(n+1)}\left\{\frac{\Gamma\left(2-\alpha+\frac{\alpha(3-\beta)}{\beta}+\frac{n \alpha}{\beta}\right)}{\Gamma\left(2+\frac{\alpha(3-\beta)}{\beta}-\frac{n \alpha}{\beta}\right)} a_{n}\right. \\
& +\xi^{-\beta} \sum_{k=0}^{n} \frac{\Gamma(1-\beta+n-k)}{1-n-k} a_{k} a_{n-k} \\
& \left.-3 \sum_{k=0}^{n}(n-k+2)(n-k+1) a_{k} a_{n-k} a_{n-k+2}\right\} x t^{-\frac{(n+3) \alpha}{\beta}} .
\end{aligned}
$$

In Figures 1-3, we have illustrated the physical features for Eq. (45) with different parameter values.

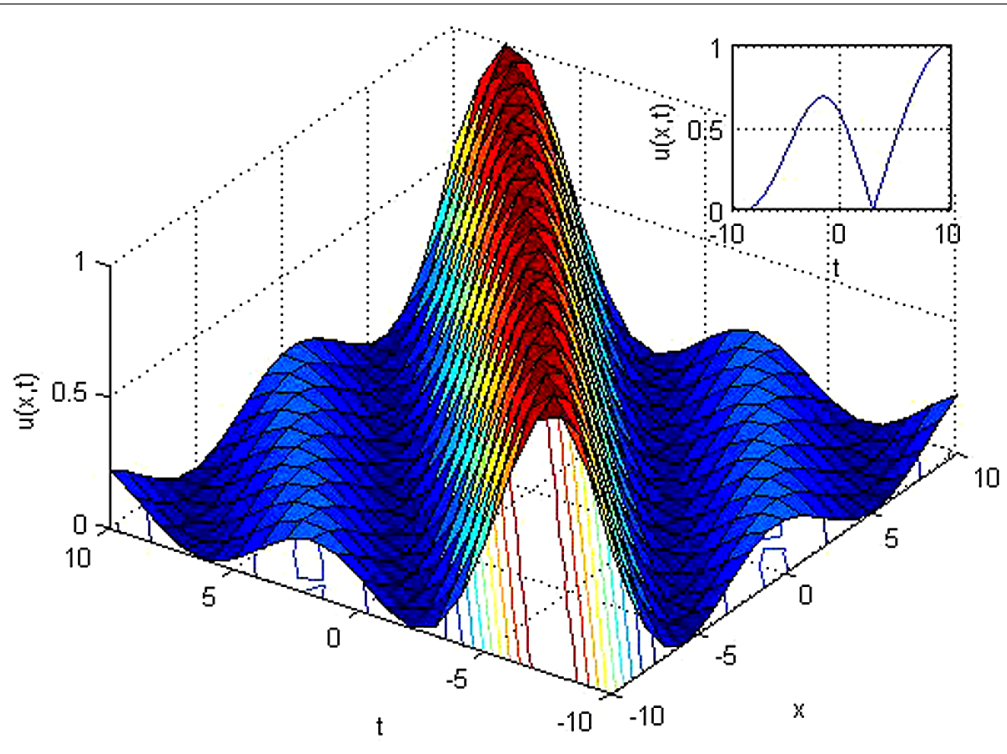

Figure 1 Numerical simulation for the 3D plot of (45). When $a_{0}=a_{1}=a_{2}=6, a_{3}=0.5, a_{4}=1.7, \beta=1.9$, $\alpha=0.5, \Gamma=0.85$. 


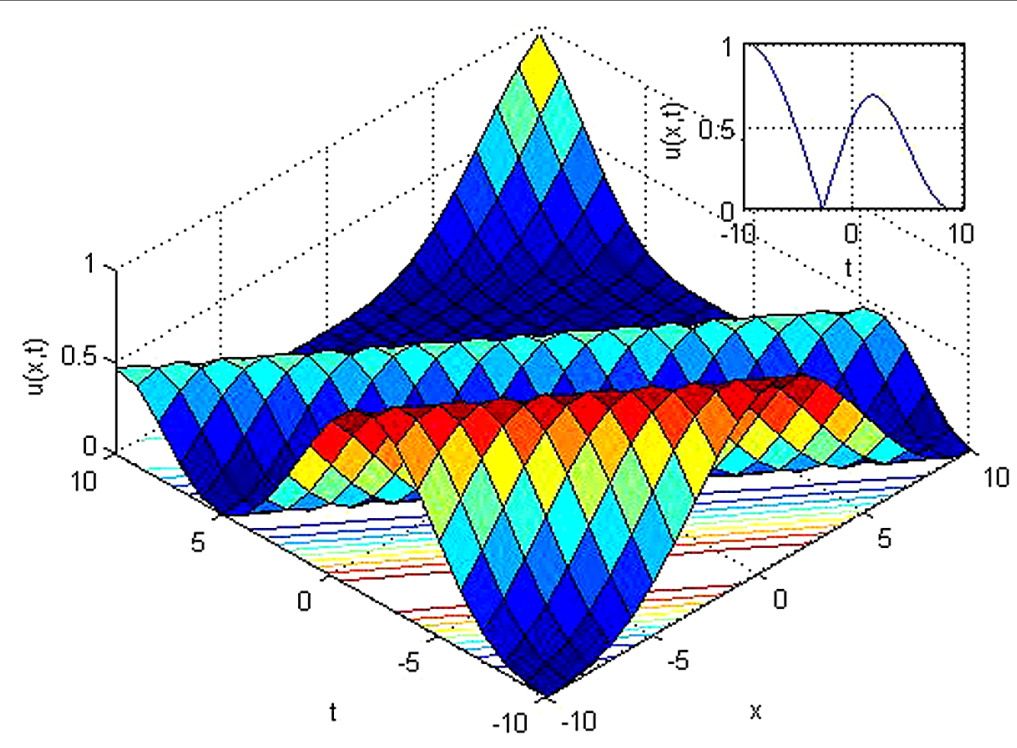

Figure 2 Numerical simulation for the 3D plot of (45). When $a_{0}=a_{1}=a_{2}=2.6, a_{3}=1.25, a_{4}=1.7$, $\beta=1.2, \alpha=0.85, \Gamma=0.5$.

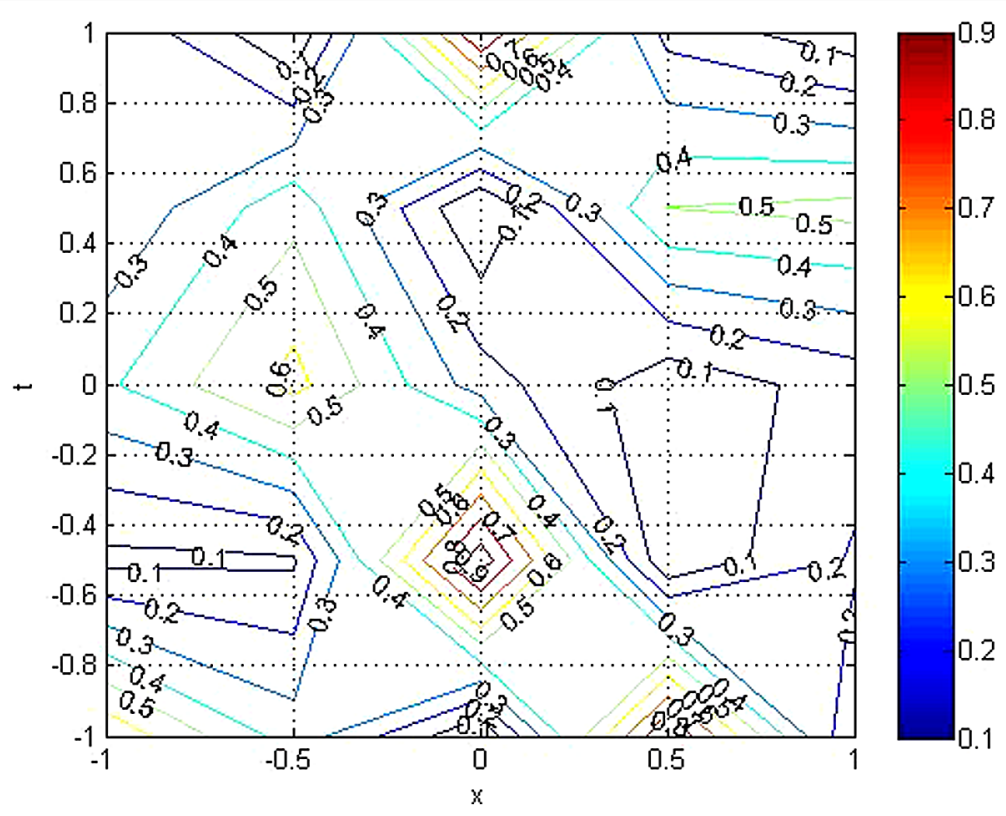

Figure 3 Numerical simulation for the contour plot of (45). When $a_{0}=a_{1}=a_{2}=2.6, a_{3}=1.25, a_{4}=1.7$, $\beta=1.5, \alpha=0.85, \Gamma=0.5$.

\section{Conservation laws}

More details about the description of Cls can be found in [1, 27, 33, 52]. The Lagrangian is presented by

$$
L=v(x, t)\left(\frac{\partial^{\alpha} u}{\partial t^{\alpha}}+u \frac{\partial^{\beta} u}{\partial t^{\beta}}+u u_{x x x}+3 u_{x} u_{x x}\right) .
$$


In Eq. (46) $v(x, t)$ is another dependent variable. The Euler-Lagrange operator [33] is

$$
\frac{\delta}{\delta u}=\frac{\partial}{\partial u}+\left(D_{t}^{\alpha}\right)^{*} \frac{\partial}{\partial D_{t}^{\alpha} u}+\left(D_{x}^{\beta}\right)^{*} \frac{\partial}{\partial D_{x}^{\beta} u}-D_{x} \frac{\partial}{\partial u_{x}}+D_{x x}^{2} \frac{\partial}{\partial u_{x x}}-D_{x x x}^{3} \frac{\partial}{\partial u_{x x x}}
$$

where $\left(D_{t}^{\alpha}\right)^{*}$ and $\left(D_{t}^{\beta}\right)^{*}$ are the adjoint operators of $\left(D_{t}^{\alpha}\right)$ and $\left(D_{t}^{\beta}\right)$, respectively. The adjoint equation is given by [33]

$$
F^{*}=\frac{\delta L}{\delta u}=0
$$

So, we can have

$$
\bar{X}+D_{t}\left(\xi^{2}\right) l+D_{x}\left(\xi^{1}\right) l=W \frac{\delta}{\delta u}+D_{t} N^{t}+D_{x} N^{x}
$$

In Eq. (49) $l$ represents the identity operator, $\frac{\delta}{\delta u}$ is the Euler-Lagrangian operator, $N^{t}$ and $N^{x}$ represent the Noether operators, $\bar{X}$ is defined by

$$
\begin{aligned}
\bar{X}= & \xi^{2} \frac{\partial}{\partial t}+\xi^{1} \frac{\partial}{\partial x}+\eta \frac{\partial}{\partial u}+\eta^{\alpha, t} \frac{\partial}{\partial D_{t}^{\alpha} u}+\eta^{\beta, x} \frac{\partial}{\partial D_{x}^{\beta} u} \\
& +\eta^{x} \frac{\partial}{\partial u_{x}}+\eta^{x x} \frac{\partial}{\partial u_{x x}}+\eta^{x x x} \frac{\partial}{\partial u_{x x x}} .
\end{aligned}
$$

With the help of Eqs. (46) and (48), we can write the adjoint equation to Eq. (1) as follows:

$$
F^{*}=\left(D_{t}^{\alpha}\right)^{*} v+u\left(D_{x}^{\beta}\right)^{*} v+u\left(v_{x}+v_{x x x}\right)=0 .
$$

Next, we look for the differential substitution for nonlinear self-adjointness. Let

$$
v=\phi(x, t, u), \quad \text { such that } \phi(x, t, u) \neq 0 .
$$

Thus, taking the derivatives of Eq. (52), we have

$$
\begin{aligned}
& v_{x}=\phi_{x}+\phi_{u} u_{x}, \\
& v_{x x}=\phi_{x x}+2 \phi_{x u} u_{x}+\phi_{u} u_{x x}+\phi_{u u} u_{x}^{2}, \\
& v_{x x x}=\phi_{x x x}+3 \phi_{x x u} u_{x}+3 \phi_{x u u} u_{x}^{2}+3 \phi_{x u} u_{x x}+3 \phi_{u u} u_{x} u_{x x}+\phi_{u} u_{x x x}+\phi_{u u u} u_{x}^{3} .
\end{aligned}
$$

Inserting Eqs. (52) and (53) into Eq. (51) and solving the results, we obtain

$$
\phi(x, t, u)=A_{1} x+A_{2} .
$$

Therefore, the variable $v(x, t)=A_{1} x+A_{2}$, and it will be used for the analysis of the Cls.

The Lie characteristic function $W$ is given by

$$
W=\eta-\xi^{2} u_{t}-\xi^{1} u_{x}
$$


The $t$ component of the conserved vectors is defined by $[33,52]$

$$
N^{t}=\xi^{2} l+\sum_{k=0}^{n-1}(-1)^{k} o D^{\alpha-1-k}(W) D_{t}^{k} \frac{\partial}{\partial o D_{t}^{\alpha} u}-(-1)^{n} J\left(W, D_{t}^{n} \frac{\partial}{\partial o D_{t}^{\alpha} u}\right),
$$

where $n=[\alpha]+1$ and $J$ is given by

$$
J(f, g)=\frac{1}{\Gamma(n-\alpha)} \int_{0}^{t} \int_{t}^{p} \frac{f\left(\xi^{2}, x\right) g(x, \mu)}{\left(\mu-\xi^{2}\right)^{\alpha+1-n}} d \mu d t
$$

Equivalently, the $x$ component of the conserved vectors $(\mathrm{CV})$ can be expressed as

$$
N^{x}=\xi^{1} l+\sum_{k=0}^{m-1}(-1)^{k} o D^{\beta-1-k}(W) D_{x}^{k} \frac{\partial}{\partial o D_{x}^{\beta} u}-(-1)^{m} J_{1}\left(W, D_{x}^{m} \frac{\partial}{\partial o D_{x}^{\beta} u}\right),
$$

where $m=[\beta]+1$ and $J_{1}$ is given by

$$
J_{1}(f, g)=\frac{1}{\Gamma(n-\beta)} \int_{0}^{x} \int_{t}^{q} \frac{f\left(\xi^{1}, t\right) g(\mu, t)}{\left(\mu-\xi^{1}\right)^{\beta+1-m}} d \mu d t .
$$

The invariance condition for any given generator $X$ of Eq. (1) and its solutions reads as follows:

$$
\left.\left(\bar{X} L+D_{t}\left(\xi^{2}\right) L+D_{x}\left(\xi^{1}\right) L\right)\right|_{\mathrm{Eq} \cdot(1)}=0
$$

and consequently, the Cls of Eq. (1) can be written as

$$
D_{t}\left(N^{t} L\right)+D_{x}\left(N^{x} L\right)=0 .
$$

Now, we present the Cls for Eq. (1) using the basic definitions presented above. Consider the following cases.

Case 1. For $\alpha \in(0,1)$, the $t$ components of the CV are

$$
\begin{aligned}
C_{1}^{t}= & o D_{t}^{\alpha-1}\left(W_{i}\right)\left(A_{1} x+A_{2}\right)+J\left(W_{i}, v_{t}\right), \\
C_{2}^{t}= & W_{i}\left(3 u_{x x}\left(A_{1} x+A_{2}\right)-3 D_{t}\left(u_{x}\left(A_{1} x+A_{2}\right)\right)+D_{t}^{2}\left(u\left(A_{1} x+A_{2}\right)\right)\right) \\
& +D_{t}\left(W_{i}\right)\left(3 u_{x}-D_{t}\left(u\left(A_{1} x+A_{2}\right)\right)\right)+D_{t}^{2}\left(W_{i} u\left(A_{1} x+A_{2}\right)\right),
\end{aligned}
$$

where $i=1,2$ and the functions $W_{i}$ are given by

$$
W_{1}=u_{x}, \quad W_{2}=\alpha u(3-\beta)-\beta t u_{t}+\alpha x u_{x} .
$$

Case 2. For $\alpha \in(1,2)$, the $t$ components of the CV are

$$
\begin{aligned}
C_{3}^{t}= & o D_{t}^{\alpha-1}\left(W_{i}\right)\left(A_{1} x+A_{2}\right)+J\left(W_{i}, v_{t}\right)-v_{t} o D_{t}^{\alpha-2}\left(W_{i}\right)-J\left(W_{i}, v_{t t}\right), \\
C_{4}^{t}= & W_{i}\left(3 u_{x x} v-3 D_{t} u_{x}\left(A_{1} x+A_{2}\right)+D_{t}^{2}\left(u\left(A_{1} x+A_{2}\right)\right)\right) \\
& +D_{t}\left(W_{i}\right)\left(3 u_{x}-D_{t}(u v)\right)+D_{t}^{2}\left(W_{i}\right) u\left(A_{1} x+A_{2}\right),
\end{aligned}
$$


where $i=1,2$ and the functions $W_{i}$ are given by

$$
W_{1}=u_{x}, \quad W_{2}=\alpha u(3-\beta)-\beta t u_{t}+\alpha x u_{x} .
$$

Case 3. For $\beta \in(0,1)$, the $x$ components of the $\mathrm{CV}$ are

$$
\begin{aligned}
C_{1}^{x}= & o D_{x}^{\beta-1}\left(W_{i}\right)\left(A_{1} x+A_{2}\right)+J\left(W_{i}, v_{t}\right), \\
C_{2}^{x}= & W_{i}\left(3 u_{x x}\left(A_{1} x+A_{2}\right)-3 D_{x} u_{x}\left(A_{1} x+A_{2}\right)+D_{x}^{2} u\left(A_{1} x+A_{2}\right)\right) \\
& +D_{x}\left(W_{i}\right)\left(3 u_{x}-D_{x} u\left(A_{1} x+A_{2}\right)\right)+D_{x}^{2}\left(W_{i}\right) u\left(A_{1} x+A_{2}\right),
\end{aligned}
$$

where $i=1,2$ and the functions $W_{i}$ are given by

$$
W_{1}=u_{x}, \quad W_{2}=\alpha u(3-\beta)-\beta t u_{t}+\alpha x u_{x} .
$$

Case 4. For $\beta \in(1,2)$, the $x$ components of the $\mathrm{CV}$ are

$$
\begin{aligned}
C_{3}^{x}= & o D_{x}^{\beta-1}\left(W_{i}\right)\left(A_{1} x+A_{2}\right)+J\left(W_{i}, v_{t}\right)-v_{t} o D_{x}^{\beta-2}\left(W_{i}\right)-J\left(W_{i}, v_{t t}\right), \\
C_{4}^{x}= & W_{i}\left(3 u_{x x}\left(A_{1} x+A_{2}\right)-3 D_{x} u_{x}\left(A_{1} x+A_{2}\right)+D_{x}^{2} u\left(A_{1} x+A_{2}\right)\right) \\
& +D_{x}\left(W_{i}\right)\left(3 u_{x}-D_{x} u\left(A_{1} x+A_{2}\right)\right)+D_{x}^{2}\left(W_{i}\right) u\left(A_{1} x+A_{2}\right),
\end{aligned}
$$

where $i=1,2$ and the functions $W_{i}$ are given by

$$
W_{1}=u_{x}, \quad W_{2}=\alpha u(3-\beta)-\beta t u_{t}+\alpha x u_{x}
$$

\section{Concluding remarks}

This work used the extension presented in $[1,2]$ to examine the LSA and Cls for the space-time fractional Rosenou-Haynam equation (RHE) with RL derivative. The spacetime fractional RHE was a reduced to space-time ODE of fractional order using its Lie point symmetries with a new dependent variable. We solved the reduced space-time ODE using the power series method for the first time. Moreover, we constructed Cls for the governing equation using a new conservation theorem.

\section{Acknowledgements}

Not applicable.

\section{Competing interests}

The authors declare that they have no competing interests.

Authors' contributions

All authors contributed equally to the writing of this paper. All authors read and approved the manuscript.

\section{Author details}

'Department of Mathematics, Cankaya University, Öğretmenler Cad., Ankara, 1406530, Turkey. ${ }^{2}$ Institute of Space

Sciences, Magurele, Bucharest, Romania. ${ }^{3}$ Department of Mathematics, Firat University, Elaziğ, 23119, Turkey.

\section{Publisher's Note}

Springer Nature remains neutral with regard to jurisdictional claims in published maps and institutional affiliations. 


\section{References}

1. Singla, K, Gupta, RK: Space-time fractional nonlinear partial differential equations: symmetry analysis and conservation laws. Nonlinear Dyn. 89(1), 321-331 (2017). https://doi.org/10.1007/s11071-017-3456-7

2. Singla, K, Gupta, RK: On invariant analysis of space-time fractional nonlinear systems of partial differential equations. II. J. Math. Phys. 58, 051503 (2017)

3. Diethelm, K: The Analysis of Fractional Differential Equations. Springer, Berlin (2010)

4. Miller, KS, Ross, B: An Introduction to the Fractional Calculus and Fractional Differential Equations. Wiley, New York (1993)

5. Podlubny, I: Fractional Differential Equations. Academic Press, San Diego (1999)

6. Oldham, KB, Spanier, J: The Fractional Calculus. Academic Press, San Diego (1974)

7. Kiryakova, V: Generalised Fractional Calculus and Applications. Pitman Res. Notes in Math., vol. 301 (1994)

8. Pinto, CMA: Novel results for asymmetrically coupled fractional neurons. Acta Polytech. Hung. 14(1), 177-189 (2017)

9. Pinto, CMA: Persistence of low levels of plasma viremia and of the latent reservoir in patients under ART: a fractional-order approach. Commun. Nonlinear Sci. Numer. Simul. 43, 251-260 (2017)

10. Carvalho, A, Pinto, CMA: A delay fractional order model for the co-infection of malaria and HIV/AIDS. Int. J. Dyn. Control 5(1), 168-186 (2017)

11. Gazizov, RK, Kasatkin, AA: Construction of exact solutions for fractional order differential equations by the invariant subspace method. Comput. Math. Appl. 66, 576-584 (2013)

12. Odibat, Z, Momani, S: A generalized differential transform method for linear partial differential equations of fractional order. Appl. Math. Lett. 21, 194-199 (2008)

13. He, TH: A coupling method of a homotopy technique and a perturbation technique for nonlinear problems. Int. J. Non-Linear Mech. 35, 37-43 (2000)

14. Wu, G, Lee, EWM: Fractional variational iteration method and its application. Phys. Lett. A 374, 2506-2509 (2010)

15. Zhang, S, Zhang, HQ: Fractional sub-equation method and its applications to nonlinear fractional PDEs. Phys. Lett. A $375,1069-1073(2011)$

16. Guo, S, Mei, LQ, Li, Y, Sun, YF: The improved fractional sub-equation method and its applications to the space-time fractional differential equations in fluid mechanics. Phys. Lett. A 376, 407-411 (2012)

17. Lu, B: Bäcklund transformation of fractional Riccati equation and its applications to nonlinear fractional partial differential equations. Phys. Lett. A 376, 2045-2048 (2012)

18. Jumarie, G: Modified Riemann-Liouville derivative and fractional Taylor series of non differentiable functions further results. Comput. Math. Appl. 51, 1367-1376 (2006)

19. Jumarie, G: Cauchy's integral formula via the modified Riemann-Liouville derivative for analytic functions of fractional order. Appl. Math. Lett. 23, 1444-1450 (2010)

20. Wang, GW, Liu, XQ, Zhang, YY: Lie symmetry analysis to the time fractional generalized fifth order KdV equation. Commun. Nonlinear Sci. Numer. Simul. 18, 2321-2326 (2013)

21. Gazizov, RK, Kasatkin, AA, Lukashchuk, Y: Continuous transformation groups of fractional differential equations. Vestn. USATU 9, 125-135 (2007)

22. Gazizov, RK, Kasatkin, AA, Lukashchuk, SY: Symmetry properties of fractional diffusion equations. Phys. Scr. 136 $014016(2009)$

23. Wang, XB, Tian, SF, Qin, CY, Zhang, TT: Lie symmetry analysis, conservation laws and exact solutions of the generalized time fractional Burgers equation. Europhys. Lett. 114, 20003 (2016)

24. Ma, PL, Tian, SF, Zhang, TT: On symmetry-preserving difference scheme to a generalized Benjamin equation and third-order Burgers equation. Appl. Math. Lett. 50, 146-152 (2015)

25. Tu, JM, Tian, SF, Xu, MJ, Zhang, TT: On Lie symmetries, optimal systems and explicit solutions to the Kudryashov-Sinelshchikov equation. Appl. Math. Comput. 275, 345-352 (2016)

26. Tian, SF, Yufeng, Z, Binlu, F, Hongqing, Z: On the Lie algebras, generalized symmetries and Darboux transformations of the fifth-order evolution equations in shallow water. Chin. Ann. Math. 36(4), 543-560 (2015)

27. Singla, K, Gupta, RK: On invariant analysis of some time fractional nonlinear systems of partial differential equations. I. J. Math. Phys. 57, 101504 (2016)

28. Olver, PJ: Applications of Lie Groups to Differential Equations. Springer, New York (1993)

29. Ibragimov, $\mathrm{NH}$, Avdonin, ED: Nonlinear selfadjointness, conservation laws, and the construction of solutions of partial differential equations using conservation laws. Russ. Math. Surv. 68, 889-921 (2013)

30. Lukashchuk, SY: Conservation laws for time-fractional subdiffusion and diffusion-wave equations. Nonlinear Dyn. 80, 791-802 (2015)

31. Frederico, GSF, Torres, DEM: A formulation of Noethers theorem for fractional problems of the calculus of variations. J. Math. Anal. Appl. 334, 834-846 (2007)

32. Atanackovic, TM, Konjik, S, Pilipovic, S, Simic, S: Variational problems with fractional derivatives: invariance conditions and Nöethers theorem. Nonlinear Anal. 71, 1504-1517 (2009)

33. Ibragimov, NH: A new conservation theorem. J. Math. Anal. Appl. 333, 311-328 (2007)

34. Wang, G, Kara, AH, Fakhar, K: Symmetry analysis and conservation laws for the class of time-fractional nonlinear dispersive equation. Nonlinear Dyn. 82, 281-287 (2015)

35. Rui, W, Xiangzhi, Z: Lie symmetries and conservation laws for the time fractional Derrida-Lebowitz-Speer-Spohn equation. Commun. Nonlinear Sci. Numer. Simul. 34, 38-44 (2016)

36. Gazizov, RK, Ibragimov, NH, Lukashchuk, SY: Nonlinear self-adjointness, conservation laws and exact solutions of time-fractional Kompaneets equations. Commun. Nonlinear Sci. Numer. Simul. 23, 153-163 (2015)

37. Qin, CY, Tian, SF, Wang, XB, Zhang, TT: Lie symmetries, conservation laws and explicit solutions for time fractional Rosenau haynam equation. Commun. Theor. Phys. 67, 157-165 (2017)

38. Rosenau, P, Hyman, JM: Compactons. Solitons with finite wavelength. Phys. Rev. Lett. 70, 564-567 (1993)

39. Yulita Molliq, R, Noorani, MSM: Solving the fractional Rosenau-Hyman equation via variational iteration method and homotopy perturbation method. Int. J. Differ. Equ. 2012, 472030 (2012)

40. Wang, XB, Tian, SF, Qin, CY, Zhang, TT: Dynamics of the breathers, rogue waves and solitary waves in the $(2+$ 1)-dimensional Ito equation. Appl. Math. Lett. 68, 40-47 (2017) 
41. Wang, XB, Tian, SF, Xu, MJ, Zhang, TT: On integrability and quasi-periodic wave solutions to a $(3+1)$-dimensional generalized KdV-like model equation. Appl. Math. Comput. 283, 216-233 (2016)

42. Feng, LL, Tian, SF, Wang, XB, Zhang, TT: Rogue waves, homoclinic breather waves and soliton waves for the image-dimensional B-type Kadomtsev-Petviashvili equation. Appl. Math. Lett. 65, 90-97 (2017)

43. Tu, JM, Tian, SF, Xu, MJ, Ma, PL, Zhang, TT: On periodic wave solutions with asymptotic behaviors to a image-dimensional generalized B-type Kadomtsev-Petviashvili equation in fluid dynamics. Comput. Math. Appl. 72, 2486-2504 (2016)

44. Xu, MJ, Tian, SF, Tu, JM, Zhang, TT: Bäcklund transformation, infinite conservation laws and periodic wave solutions to a generalized (2+1)-dimensional Boussinesq equation. Nonlinear Anal., Real World Appl. 31, 388-408 (2016)

45. Wang, XB, Tian, SF, Qin, CY, Zhang, TT: Characteristics of the solitary waves and rogue waves with interaction phenomena in a generalized (image)-dimensional Kadomtsev-Petviashvili equation. Appl. Math. Lett. 72, 58-64 (2017)

46. Wang, XB, Tian, SF, Yan, H, Zhang, TT: On the solitary waves, breather waves and rogue waves to a generalized (3+ 1)-dimensional Kadomtsev-Petviashvili equation. Comput. Math. Appl. 74, 556-563 (2017)

47. Tu, JM, Tian, SF, Xu, MJ, Zhang, TT: Quasi-periodic waves and solitary waves to a generalized KdV-Caudrey-Dodd-Gibbon equation from fluid dynamics. Taiwan. J. Math. 20, 823-848 (2016)

48. Wang, GW, Xu, TZ: Invariant analysis and exact solutions of nonlinear time fractional Sharma-Tasso-Olver equation by Lie group analysis. Nonlinear Dyn. 76, 571-580 (2014)

49. Luchko, YU, Gorenflo, R: Scale-invariant solutions of a partial differential equation of fractional order. Fract. Calc. Appl. Anal. 1, 63-78 (1998)

50. Hilfer, R: Applications of Fractional Calculus in Physics. World Scientific, Singapore (2000)

51. Galaktionov, VA, Svirshchevskii, SR: Exact Solutions and Invariant Subspaces of Nonlinear Partial Differential Equations in Mechanics and Physics. Chapman \& Hall, Boca Raton (2006)

52. Noether, E: Invariant variation problems. Transp. Theory Stat. Phys. 1(3), 186-207 (1971)

\section{Submit your manuscript to a SpringerOpen ${ }^{\circ}$ journal and benefit from:}

- Convenient online submission

- Rigorous peer review

- Open access: articles freely available online

- High visibility within the field

Retaining the copyright to your article

Submit your next manuscript at $\gg$ springeropen.com 